\title{
Householder-based Prony Method for Identification of Low- frequency Oscillation in Power System
}

\author{
Simin Zhang ${ }^{1}$, Guangye Liu ${ }^{1, *}$, Yaxin $\mathrm{Li}^{1}$, and Jiangang Yao ${ }^{1}$ \\ ${ }^{1}$ College of Electrical and Information Engineering, Hunan University, Changsha 410082, China
}

\begin{abstract}
In this paper, the Prony analysis tool with the software used in the actual operation has a large error. The improved Prony method is used to identify the low-frequency oscillation mode of the power system. The traditional Prony algorithm has the problem of insensitivity to parameter error estimation in low frequency oscillation, which affects the accuracy and accuracy of the least squares calculation. Therefore, this paper proposes a Prony method power system low frequency oscillation method using Householder transform to make the calculation more Accurate and proven to be more efficient and reliable.
\end{abstract}

\section{Introduction}

With the continuous development of intelligent largescale power grids, power systems include water, fire, nuclear, wind, light and other forms of power supply. In some areas, small hydropower is enriched, mixed with $\mathrm{AC}$ and $\mathrm{DC}$, and connected with local power and exclusive power grids. The structure and characteristics of the grid determine that the dynamic stability of the power grid will exist for a long time, and low-frequency oscillations occur from time to time. It is still an important aspect that affects system security and restricts system power transmission. The dynamic stability of the power grid analysis is based on the PSD-SSAP power system small interference stability analysis program for frequency analysis, using PSD-BPA electromechanical transient simulation software for time domain simulation analysis [1], but the prony toolbox analysis error Larger, theoretically, a more accurate analysis method for lowfrequency oscillations is needed. There are many methods for low-frequency oscillation, such as eigenvalue analysis, wavelet analysis, Fu-style algorithm, and time-domain simulation. The Prony algorithm, which appeared in 1795 , uses a linear combination of exponential functions to describe the mathematical model of equally spaced sampled data, which can estimate the frequency, attenuation factor, amplitude, and initial phase of a given signal [2].

Although the current Prony algorithm is a common method for studying low-frequency oscillations in power systems, it can extract the oscillation characteristics more accurately and find the dominant oscillation mode [3]. However, in practical applications, the Prony algorithm has problems such as time window selection, calculation accuracy, and noise sensitivity [4]. For the selection of time window, the literature [5] proposed to adopt sliding window processing for signals; on the basis of this, the literature [6] proposes to reduce the influence of noise on the basis of sub-window data processing. The literature [7] proved that the Givens segmentation method divides the sampling time into two time segments, and proposes different attenuation solutions for the slow and fast two different attenuations, which solves the problem of losing the attenuation component in the low frequency oscillation. If there is too much data to cause overflow, the literature [8] proposes a method of segmentation average compression filtering, which does not require SVD singular value calculation. In the fixed order, the literature [9] proposed an improved smoothing priority method. After ISPM filtering, the high frequency noise is quickly and accurately removed, and SDMProny identification is performed to obtain the dominant mode of low frequency oscillation. The method is based on singular value decomposition. The literature[10] proposed a mathematical method is proposed to recursively complex parameter least squares estimation, which makes the calculation accuracy higher. It is considered to be used in the traditional Prony algorithm for correlation optimization.

The dynamic stability of the power grid has always been a concern. At present, the energy Internet is developing rapidly, and the stability of the power grid is higher. Therefore, the low frequency oscillation of the original Prony algorithm is optimized. In this paper, the QR decomposition method of Householder transformation is used to improve the fitting precision. The improvement of this algorithm is helpful and be used in the analysis of actual low frequency oscillation actual work through the comparison of examples.

The main contents of the remainder of this paper are as follows: The second part introduces the traditional Prony algorithm and proposes the need for improvement; the third part focuses on how to combine the Householder transform with the traditional algorithm to get an improved algorithm; the fourth part of the case

\footnotetext{
* Corresponding author: liuguangye@hnu.edu.cn
} 
Perform calculations and analysis; the fifth part is summary and outlook.

\section{Principle of the Prony algorithm}

The Prony algorithm is a linear combination of a set of exponential functions to simulate the real sampled signal, and the amplitude, phase, frequency, attenuation factor and other information of the model can be obtained [11]. Its discrete time function relationship is:

$$
\hat{x}(t)=\sum_{i=1}^{p} b_{i} z_{i}^{t} \quad t=0,1, \cdots, T-1
$$

Where $x(t)$ is an approximation of the sampled data.

We define the sampled signal as attenuated sinusoidal component whose function expression is:

$$
x(t)=\sum_{i=1}^{Q} A_{i} e^{\alpha_{i} t} \cos \left(2 \pi f_{i} t+\theta_{i}\right)
$$

In the above formula, $A_{i}$ is the amplitude; $\theta_{i}$ is the initial phase; $\alpha_{i}$ is the oscillation frequency ( $\left.\mathrm{Hz}\right) ; \alpha_{i}$ is the attenuation factor (damping); $Q$ is the order.

The difference equation obtained by equation (2) is:

$$
\hat{x}(t)=-\sum_{k=1}^{p} a_{k} \hat{x}(n-k) \quad n=0,1, \cdots, N-1
$$

Define:

$$
r(i, m)=\sum_{n=p}^{N-1} x(n-m) x^{*}(n-i)
$$

The Prony algorithm equation can be obtained as:

$$
\left(\begin{array}{cccc}
r(0,0) & r(0,1) & \cdots & r\left(0, p_{e}\right) \\
r(1,0) & r(1,1) & & r\left(1, p_{e}\right) \\
\vdots & \vdots & & \vdots \\
r\left(p_{e}, 0\right) & r\left(p_{e}, 1\right) & \cdots & r\left(p_{e}, p_{e}\right)
\end{array}\right)\left(\begin{array}{c}
1 \\
\alpha_{1} \\
\vdots \\
\alpha_{p}
\end{array}\right)=\left(\begin{array}{c}
\varepsilon_{p} \\
0 \\
\vdots \\
0
\end{array}\right)
$$

Find the eigenvalue according to equation (5):

$$
1+\alpha_{1} z^{-1}+\cdots+\alpha_{p} z^{-p}=0
$$

The linear equation for $b_{i}$ can be expressed as:

$$
\left(\begin{array}{cccc}
1 & 1 & \cdots & 1 \\
z_{1} & z_{2} & \cdots & z_{p} \\
\vdots & \vdots & & \vdots \\
z_{1}^{N-1} & z_{2}^{N-1} & \cdots & z_{p}^{N-1}
\end{array}\right)\left(b_{1}, b_{2} \cdots b_{p}\right)^{T}=(\hat{x}(1), \hat{x}(2) \cdots \hat{x}(p))^{T}
$$

The expression (8) of the oscillation frequency, phase, amplitude, and attenuation factor can be obtained by calculating $b_{i}$ according to equation (7):

$$
\left\{\begin{array}{c}
f_{i}=\arctan \left[\operatorname{Im}\left(z_{i}\right) / \operatorname{Re}\left(z_{i}\right)\right] /(2 \pi \Delta t) \\
\theta_{i}=\arctan \left[\operatorname{Im}\left(b_{i}\right) / \operatorname{Re}\left(b_{i}\right)\right] \\
A_{i}=\left|b_{i}\right| \\
\alpha_{i}=\ln \left|z_{i}\right| / \Delta t
\end{array}\right.
$$

The traditional Prony algorithm calculates the order of the model by calculating the rank of the equation by equation (5), thereby obtaining the number of components after the low-frequency oscillation occurs. However, since the algorithm accuracy is not high enough, if the model order is less than the actual order, the error generated detrimental to the analytical signal. Conversely, if the order of the model is greater than the actual order, the individual components of the oscillation mode can be extracted. The method of QR decomposition least squares parameter estimation is mostly used in the real field, even if the processing of the complex signal can be converted into the real field, but the corresponding calculation time is increased. In order to quickly extract the frequency, phase, amplitude, attenuation factor and other information of low frequency oscillation, the traditional Prony algorithm needs to be improved. In the process of obtaining the eigenvalue, the least squares estimation algorithm of Householder transform is used to carry out the traditional algorithm. The original coefficient matrix is transformed into an upper triangular matrix, and the least squares estimation solution is obtained after iterative recursion.

\section{Improvement of prony algorithm}

\subsection{Householder transformation}

In the process of solving the coefficient of the characteristic equation of the traditional least squares parameter estimation algorithm, the matrix is solved by the Givens transform. Givens transform is a common method in QR transformation. In the fixed-order problem of the least-squares of the autoregressive-sliding average model, the system generates the anti-interference ability due to the excessive conditional number. In fact, it is related to the orthogonality of the basis vector[12]. If the inner product of the two vectors is zero, then it can be shown that the two vectors are orthogonal. In threedimensional space, it can be seen that the orthogonal two vectors are perpendicular to each other. If the mutually orthogonal vectors are all 1 in length, they are also called standard orthogonal bases. With the Givens rotation method, any vector can be rotated to the unit vector as long as the angle between the two coordinates is determined. The Givens rotation method needs to be completed step by step. The huge complex signal increases the calculation time. Considering the need of signal processing speed, this paper uses Householder transform to improve the speed of transformation and get a more concise and quick formula

In the calculation of the Prony algorithm by least squares fitting, there is the following formula:

$$
x(t)=\hat{x}(t)+\varepsilon(t) \quad t=0,1, \cdots N-1
$$

Substituting the difference equation (3) into equation (9) can be transformed into:

$$
x(t)=-\sum_{k=1}^{p} \alpha_{k} \hat{x}(n-k)+\varepsilon(t)
$$

Make the objective function:

$$
J(N)=\sum_{n=p}^{N-1}|\mathcal{E}(t)|^{2}=\min
$$


According to the Householder transformation, combined with the Prony algorithm, the coefficient matrix can be defined as:

$$
\boldsymbol{\alpha}(P)=\left(\begin{array}{c}
\alpha_{1} \\
\alpha_{2} \\
\vdots \\
\alpha_{p}
\end{array}\right)
$$

There is a Householder transformation Q, which transforms the equation (12) into an upper triangular matrix:

$$
\begin{gathered}
\mathbf{Q} \boldsymbol{\alpha}(P)=\left(\begin{array}{cccc}
r_{11} & r_{12} & \cdots & r_{1 p} \\
& r_{22} & \cdots & r_{2 p} \\
& & \ddots & \vdots \\
& & & r_{p p} \\
0 & \cdots & \cdots & 0 \\
\vdots & \ddots & & \vdots \\
\vdots & & \ddots & \vdots \\
0 & \cdots & \cdots & 0
\end{array}\right)=\left(\begin{array}{c}
\mathbf{R}(P) \\
0
\end{array}\right) \\
\mathbf{Q X}=\left(z_{1}, \cdots, z_{M}, z_{M+1}, \cdots z_{N}\right)^{T}=\left(p^{T}(N), v^{T}(N)\right)^{T}
\end{gathered}
$$

The least squares estimate can be recursive according to $(15)$

$$
\mathbf{R}(P) X=p(N)
$$

The objective function (11) can be reduced to equation (16):

$$
J(N)=\|v(N)\|_{2}^{2}
$$

After the recursive iteration, the Householder transform realizes the upper triangulation. The eigenvalues of the low-frequency oscillation can obtain more accurate parameter precision through subsequent algorithm analysis and comparison, which greatly improves the efficiency and accuracy of the operation.

\subsection{The analysis of Householder transformation process}

(1) Read the sampled data. In order to accurately extract the oscillation parameters, the sampling interval can be set to $0.01 \mathrm{~s}$, and a total of 1500 sample values, the sampling duration is $15 \mathrm{~s}$.

(2) The sampled data is subjected to noise reduction processing, and then filtered by an ideal filter to remove high frequency components in the signal. The chosen order should be greater than or equal to the effective rank, ensuring that each component can be extracted quickly and accurately. If the selected order is less than the effective rank, the obtained power frequency error will be large, which is not conducive to subsequent calculations. The filtered sampled data is expanded to form a corresponding matrix, and the matrix is calculated by the singular value decomposition method, the effective rank of the extended matrix is redetermined, and the order is selected to be within a reasonable range. The coefficient of the characteristic equatio is obtained by the Householder transformation, and then the eigenvalue is obtained by substituting into the characteristic equation. The value of the eigenvalue is substituted into the equation (8) to obtain the frequency and attenuation factor. From (7), the parameter can be obtained, and then substituted into the equation (8) to obtain the initial phase and the amplitude. The four parameters of the initial phase, amplitude, frequency and attenuation factor are substituted into equation (2) to obtain the expression of the signal. The effect of solving the signal with the Householder transform has been greatly improved.

\section{Case analysis}

In order to prove the parameter value of the lowfrequency oscillation of the power system low-frequency oscillation mode based on the Householder transform, the traditional Prony algorithm and the Prony method based on Householder transform can be compared and analyzed. The improved-Prony algorithm greatly improves the extraction effect of the traditional algorithm and accurately obtains the attenuation component.

Set the low frequency oscillation signal to the following formula:

$x=0.2 \times e^{-0.03 t} \times \cos (2 \pi \times 0.25 t)+0.8 \times e^{-0.08 t} \times \cos (2 \pi \times 0.7 t) \quad(17)$

The model order of the signal is uniformly taken as 5 , and the above signal has 2 sinusoidal components, that is, 4 complex exponential components. The sampling value is 1500 , the sampling interval is 0.01 , and the sampling duration is $15 \mathrm{~s}$. The component parameters of each signal are shown in Table 1.

Table 1. A case of signal parameter.

\begin{tabular}{|c|c|c|c|c|}
\hline $\begin{array}{c}\text { Compo- } \\
\text { nent } \\
\text { number }\end{array}$ & $\mathbf{f}$ & $\boldsymbol{\alpha}$ & $\mathbf{A}$ & $\boldsymbol{\theta}$ \\
\hline 1 & 0.25 & -0.03 & 0.2 & 0 \\
\hline 2 & 0.8 & -0.08 & 0.8 & 0 \\
\hline
\end{tabular}

\begin{tabular}{|c|c|c|c|c|c|}
\hline $\begin{array}{c}\text { Com } \\
\text { pone } \\
\text { nt } \\
\text { numb } \\
\text { er }\end{array}$ & $\begin{array}{c}\text { metho } \\
\text { d }\end{array}$ & f & $\boldsymbol{\alpha}$ & $\mathbf{A}$ & $\boldsymbol{\theta}$ \\
\hline \multirow{2}{*}{1} & $\begin{array}{c}\text { Traditi } \\
\text { onal } \\
\text { algorit } \\
\mathrm{hm}\end{array}$ & $\begin{array}{c}0.248 \\
9\end{array}$ & -0.0305 & 0.2 & 0 \\
\hline & $\begin{array}{c}\text { Impro } \\
\text { ved } \\
\text { algorit } \\
\mathrm{hm}\end{array}$ & 0.25 & -0.03 & 0.2 & 0 \\
\hline 2 & $\begin{array}{l}\text { Traditi } \\
\text { onal } \\
\text { algorit } \\
\mathrm{hm}\end{array}$ & $\begin{array}{c}0.708 \\
6\end{array}$ & 0.0895 & $\begin{array}{c}0.799 \\
3\end{array}$ & 0 \\
\hline
\end{tabular}

The two algorithms were simulated separately, and the comparison results are shown in Table 2.

Table 2. Comparison of results between traditional and improved algorithms. 


\begin{tabular}{|l|c|c|c|c|c|}
\hline & $\begin{array}{c}\text { Impro } \\
\text { ved } \\
\text { algorit } \\
\mathrm{hm}\end{array}$ & 0.8 & -0.08 & 0.8 & 0 \\
\hline
\end{tabular}

It can be seen that both algorithms can extract the oscillation parameter information, but the modified prony algorithm has a smaller deviation than the original prony algorithm and is closer to the theoretical value.

The graph formed by the two algorithms is shown in Fig 1. It can be more intuitively seen that compared with the traditional prony algorithm, the Householder transform is applied to the solution process of the eigenvalues, and a more accurate parameter estimation effect can be obtained. Proceedings of the 9th Power Quality Symposium.

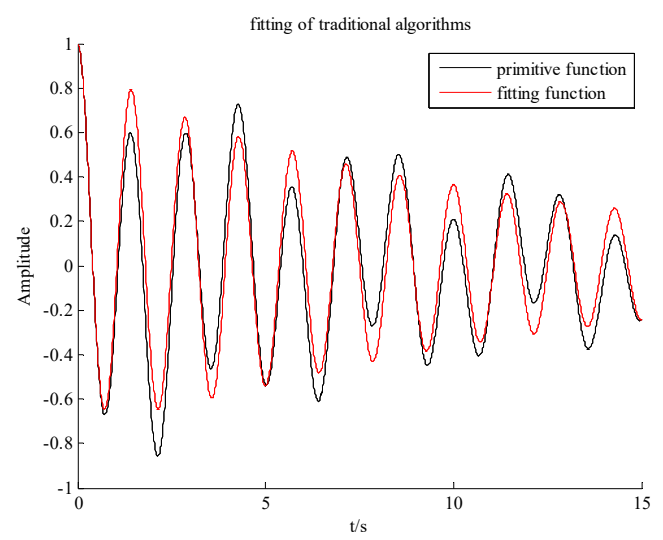

Fig. 1. Original algorithm fitting effect.

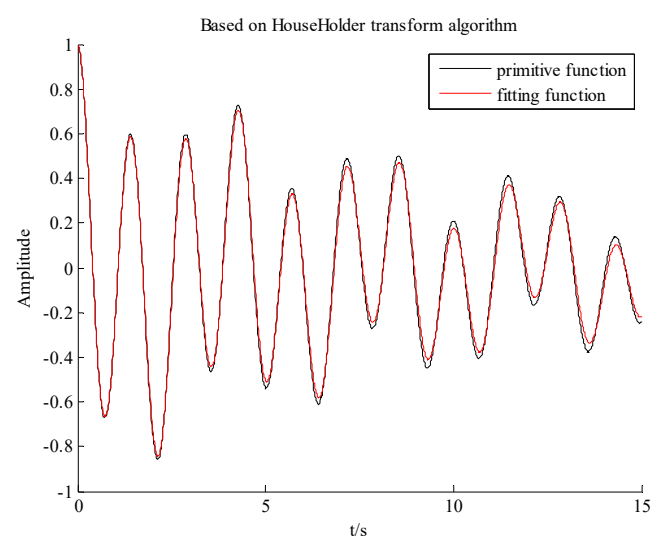

Fig. 2. Improved algorithm fitting effect.

\section{Conclusion}

The traditional Prony algorithm can accurately identify the information of the dominant oscillation mode, but the data loss will occur and affect the accuracy of the identification. The improved algorithm in this paper combines the advantages of Householder transform and Prony algorithm, and has great advantages for lowfrequency oscillation information after noise reduction processing. The example shows that the Householder transform has the effect of improving the numerical stability of the traditional algorithm. The memory of the algorithm is small, the computer calculation speed is faster, and the low-frequency oscillation component can be accurately extracted. It is effective in the practical application of low-frequency oscillation.

The improved algorithm can be used in the dynamic stability content of small interference stability analysis, and can be combined with theory and practical application. This paper does not discuss the effectiveness of the algorithm under noise interference. The subsequent work needs to improve the algorithm optimization under the interference of noise, and write the corresponding software program to be applied in the dynamic stability analysis software. It will play an important role in the decision-making of the power grid, and the security and stability will be further improved.

\section{References}

1. Y. H. Chen, Research on Low Frequency Oscillation Disturbance Source Location Based on Network Topology Data(2017)

2. T. Q. Wang, R. M. He , D. J. Xu, X. W. Wang, ELECTRC POWER, 34, 38(2001)

3. Y. B. Wang, Research on Accurate Identification Method of Fast Attenuating Component of Frequency Signal(2016)

4. Y. D. Wen, Y. P. Zhao, M. Gan, M. Ding, Journal of Hefei University of Technology 37, 1046 (2014)

5. J. F. Zhang, T. Yang, M. Chen, T. T. Zhang, J. Xiao, C. X. Mao, Eletric Power Automation Equipment 38 (2018)

6. L. Ding, A. C. Xue, J. Li, J. P. Wang, F. K. Han, M. H. Wang, Automation of Eletric Power System 34 (2010)

7. X. J. Zhang, JiangXi Eletric Power 36 (2012)

8. C. Zhang, T. Jin, Power System Technology 40 (2016)

9. B. W. Cui, Journal of Anhui University 37 (2013)

10. J. W. Mou, Y. N. Shang, W. G. Liu, S. Y. Dai, P. Jiang, B. L. Wang, Y. B. Wang, Journal of Electrical engineering 13 (2018)

11. Y. Q. Wang, H. Liu,Wireless Internet Technology 138 (2013)

12. Y. Bai, R. Huo, X. Li, Proceedings of the $9^{\text {th }}$ Power Quality Symposium 384 (2018) 